\title{
Co-occurrence of apocrine adenocarcinoma and invasive mammary-type ductal carcinoma in extramammary Paget disease of the axilla
}

\author{
Seung Bin Jang ${ }^{1}$, Sung-Eun $\mathrm{Kim}^{1}$, Young Ah Kim², Hye Ryeon Choi ${ }^{3}$ \\ Departments of ${ }^{1}$ Plastic and Reconstructive Surgery, and ${ }^{2}$ Pathology, School of Medicine, Catholic University of Daegu, Daegu; ${ }^{3}$ Department \\ of Surgery, Yonsei University College of Medicine, Seoul, Korea
}

Extramammary Paget disease (EMPD) is an uncommon malignancy that occurs in apocrine gland-rich areas of the body. EMPD of the axilla is rare, but a few cases have been reported. Some cases of EMPD have been reported with underlying apocrine adenocarcinoma; rarely, mammary-type ductal carcinoma can accompany EMPD. Here, we report a very rare case of EMPD with apocrine adenocarcinoma and invasive mammary-type ductal carcinoma. A 55-year-old woman was referred with a brownish pigmented plaque accompanying an area of ulceration in the left axilla. A preoperative biopsy indicated Paget disease, and an additional evaluation was performed to determine whether it was of primary or secondary origin. A wide excision was made, and the axilla was reconstructed using a thoracodorsal artery perforator flap. The biopsy showed apocrine adenocarcinoma and invasive mammary-type ductal carcinoma with pagetoid spreading. The patient had no evidence of recurrence or other postoperative complications.

Keywords Extramammary Paget disease / Axilla / Immunohistochemical staining
Correspondence: Sung-Eun Kim Department of Plastic and Reconstructive Surgery, School of Medicine, Catholic University of Daegu, 33 Duryugongwon-ro 17-gil, Nam-gu, Daegu 42472, Korea

Tel: +82-53-650-4578

Fax: +82-53-650-4584

E-mail: fdghfg26@cu.ac.kr

Received: September 5, 2019 • Revised: October 30, 2019 • Accepted: October 30, 2019

pISSN: 2234-6163・ elSSN: 2234-6171 • https://doi.org/10.5999/aps.2019.01172• Arch Plast Surg 2020;47:83-87

\section{INTRODUCTION}

Extramammary Paget disease (EMPD) is a rare type of intraepithelial adenocarcinoma that often affects apocrine gland-bearing areas, such as the vulva, penis, scrotum, axilla, and even the eyelids [1]. EMPD is most commonly found in the vulvar area, which accounts for $65 \%$ of all cases. Although EMPD rarely affects the axilla, cases of axillary Paget disease have been reported in the literature [2].

EMPD is generally a solitary disease that arises from multipotent cells in the epidermis and can become invasive carcinoma. EMPD can occur in combination with underlying adenocarci- noma of the skin appendages [3]. Involvement of the adnexa is a common feature of EMPD. Most cases of EMPD with underlying adnexal carcinoma are found in the anogenital area, and few have been found in the axilla. Morgan et al. [4] reported a case of Paget disease coexisting with underlying invasive apocrine carcinoma in the unilateral axilla. Very rarely, EMPD has occurred in association with an underlying mammary-like carcinoma [5]. Villada et al. [3] reported a case of EMPD of the vulva with an underlying adenocarcinoma or mammary-like carcinoma that originated from an anogenital mammary-like gland. These mammary-like carcinomas of the vulva are extremely rare, and have been observed in fewer than 5\% of EMPD cases 
[6]. Furthermore, no cases of axillary EMPD with apocrine adenocarcinoma accompanied by mammary-type carcinoma have yet been reported [4]. In this paper, we report a case of axillary EMPD accompanied by both apocrine adenocarcinoma and invasive mammary-type ductal carcinoma.

\section{CASE}

A 55-year-old woman was referred with a 2-year history of an erythematous, brownish pigmented plaque with erosion, scales, and ulceration in the left axilla (Fig. 1). Upon physical examination, the symmetry of the breasts was normal, without domi-

\section{Fig. 1. Preoperative photo}

A $7.0 \times 6.0 \mathrm{~cm}$ lesion on the left axilla with erythema, brownish pigmented plaque, erosion, and scales.

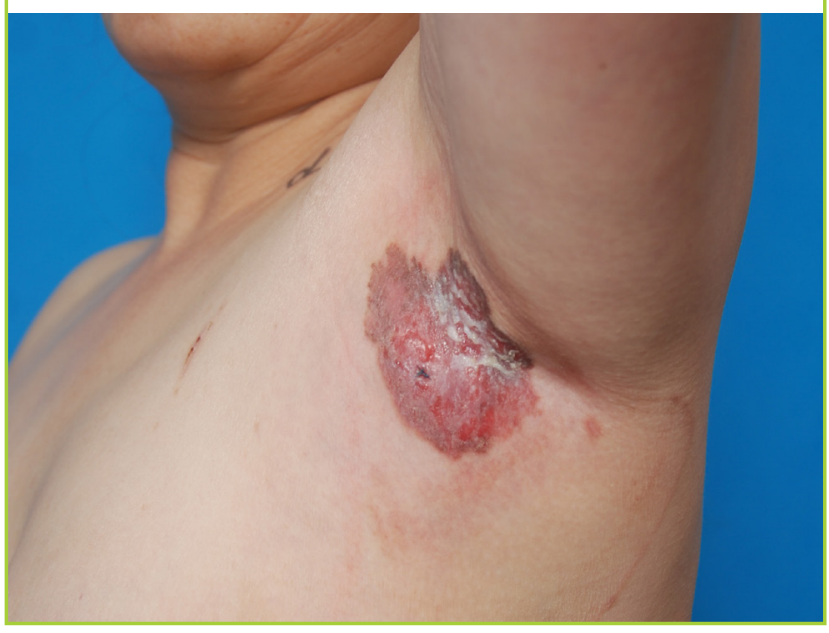

\section{Fig. 2. Skin punch biopsy}

Skin biopsy tissue shows the pagetoid spread of clear vacuolated cells in the epidermis $\left(H \& E_{1} \times 400\right)$. The tumor cells in the epidermis display polygonal nuclei with abundant cytoplasm. Some tumor cells have intracytoplasmic vacuoles (red arrow).

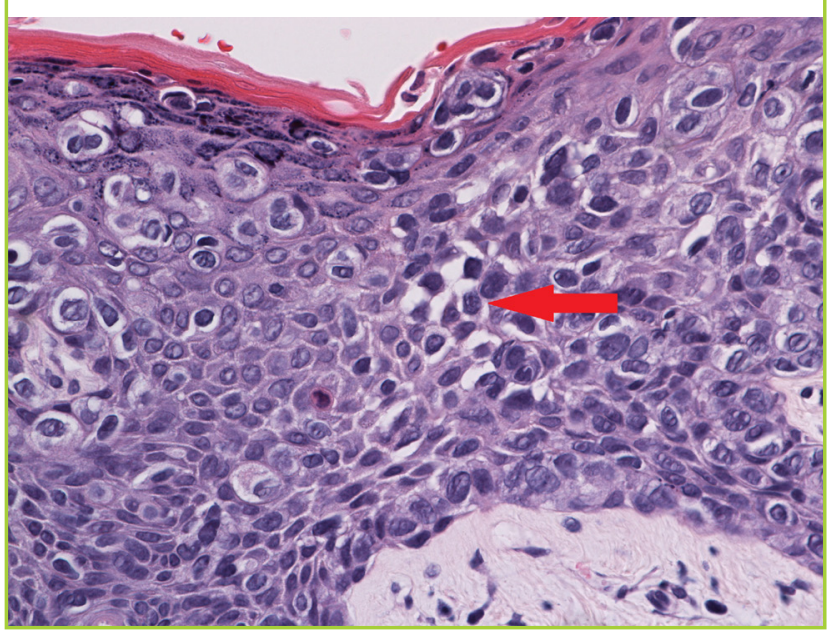

nant nodules in either breast and with no evidence of nipple inversion or discharge. No evidence of lymphadenopathy was found, and the physical examination was within normal limits.

The patient had a personal history of uterine cervix carcinoma and had undergone a hysterectomy. She had no family history of breast cancer, but her father, sister, and brother each had a history of liver cancer.

A punch biopsy performed by a dermatologist was positive for Paget disease (Fig. 2). Evaluations to determine whether it was of primary or secondary origin were conducted using mammography, breast sonography, esophagogastroduodenoscopy, and colonoscopy. Additionally, a positron emission tomography-computed tomography scan was performed to evaluate the patients for distant metastases. No primary lesion or distant metastasis was found (Fig. 3).

EMPD can be invasive. In our patient, a wide excision with 2to 3-cm margins was performed with an axillary sentinel lymph node excisional biopsy. We covered a large defect area of the axilla, using a thoracodorsal artery perforator (TDAP) flap for reconstruction (Fig. 4).

The histological findings of the excised lesion showed intraepidermal spreading of tumor cells involving the apocrine gland and perifollicular structure; additionally, neoplastic cells that displayed pagetoid spreading were noted in the epidermis (Fig. $5 \mathrm{~A}$ and $\mathrm{B}$ ). Some nested tumor cells resembling invasive ductal

\section{Fig. 3. Preoperative PET-CT scan}

PET-CT scan showing cutaneous thickening and a subcutaneous nodular lesion with heterogeneous hypermetabolism $\left(S \mathrm{VV}_{\max }=4.65\right)$ in the left axilla.

PET-CT, positron emission tomography-computed tomography; SUV max, $_{\text {, }}$ aximum standardized uptake value.

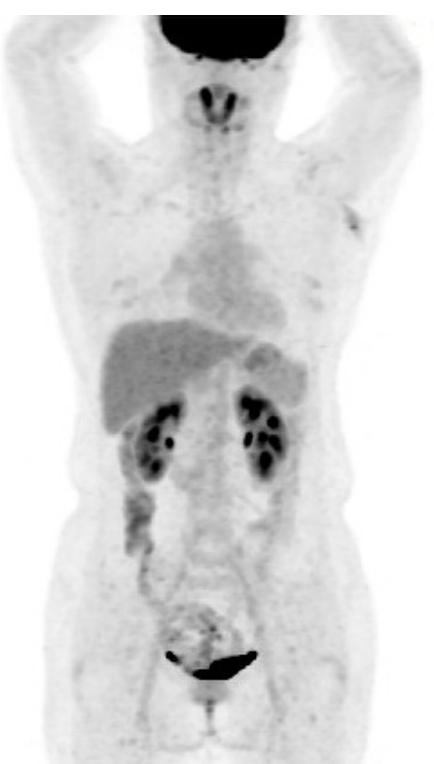


carcinoma of the breast were also noted (Fig. 5C and D).

Immunohistochemical stains showed strong positive reactivity for cytokeratin 7 , estrogen receptor, and human epidermal growth factor receptor 2 , as well as focal positive reactivity for gross cystic disease fluid protein 15 . No reactivity was noted for epidermal growth factor receptor or progesterone receptor. The excision specimen was diagnosed as apocrine adenocarcinoma and invasive mammary-type ductal carcinoma with epidermal pagetoid spreading. Although the specimen resembled breast cancer, ectopic mammary tissue was not identified.

After the operation, chemotherapy was not performed. Instead, adjuvant radiotherapy was administered as treatment for adnexal cancer. At 12 months postoperatively, the patient had no evidence of recurrence or other postoperative complications. Additionally, she had no functional problems with the range of movement of the shoulder (Fig. 4).
Fig. 4. Postoperative photography (after 12 months)

No evidence of recurrence or other postoperative complications. No functional problem with the range of movement of the shoulder.

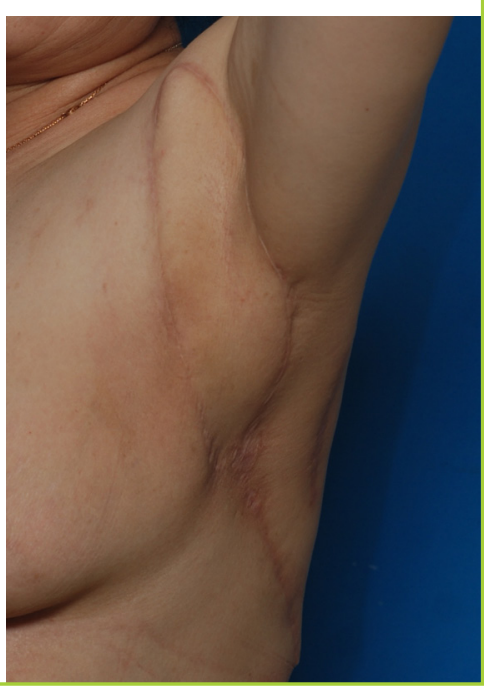

\section{Fig. 5. Histological features}

(A) The apocrine gland and perifollicular structure (red arrow) are affected by invasive carcinoma. Infiltrating tumor nests are also present (indicated by the square) $\left(H \& E_{1} \times 40\right)$. (B) Neoplastic cells exhibiting pagetoid spreading are noted in the epidermis $\left(H \& E_{1} \times 100\right)$. (C) Tumor cells display a non-glandular architecture and prominent nucleoli in variable-sized nuclei; these cells resemble high-grade invasive ductal carcinoma $\left(H \& E_{1} \times 400\right)$. (D) Some of the invasive lesion is composed of small nested or singly scattered tumor cells (red arrow) $\left(H \& E_{1} \times 400\right)$.
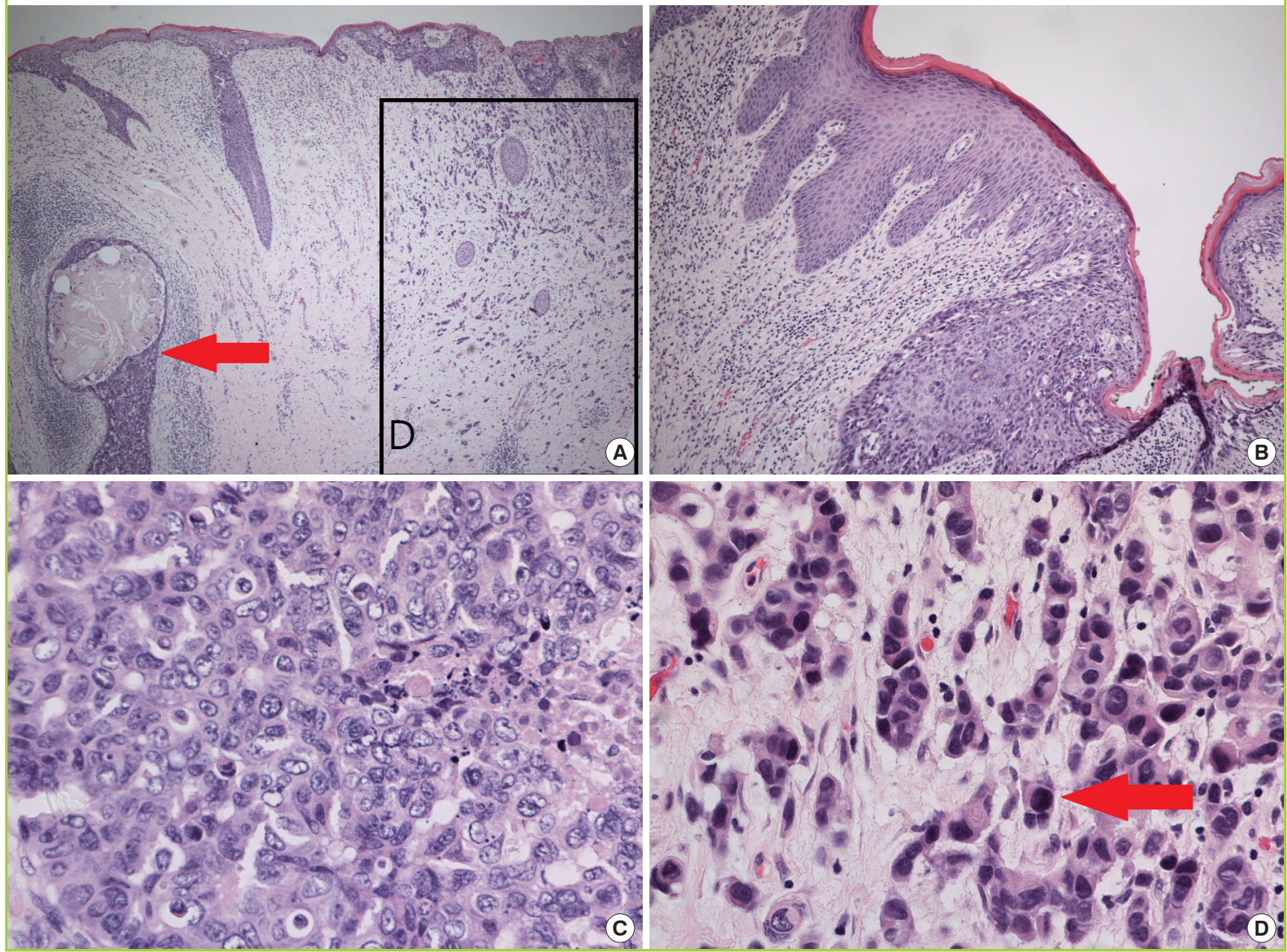


\section{DISCUSSION}

Primary EMPD and mammary Paget disease (MPD) are similar in that both involve intraepithelial proliferation of carcinoma cells and are diagnosed according to their microscopic features. However, MPD and EMPD have different characteristics. MPD is almost always associated with underlying ductal carcinomaeither in situ or invasive-or, rarely, lobular carcinoma. In contrast, the histogenesis of primary EMPD is less clear and is still the subject of debate. The skin adnexa (specifically, the apocrine or eccrine glands), vulvar Toker cells, anogenital mammary-like glands, and pluripotential germinative cells in the epidermis and other structures have been implicated as potential sources of neoplastic cells [7].

To diagnose EMPD, immunohistochemical stains, including stains for cytokeratin 7 and gross cystic disease fluid protein 15, were performed and showed positive reactivity [5]. We also performed tests for breast cancer markers, such as estrogen receptor/progesterone receptor and human epidermal growth factor receptor, because mammary-like ductal carcinoma (which resembles breast cancer) was identified through microscopy. As the tumor involved the apocrine gland, we concluded that EMPD was associated with underlying apocrine carcinoma and mammary-like ductal carcinoma.

When EMPD with apocrine carcinoma is found in the axilla, it is necessary to rule out an underlying breast carcinoma or primary breast cancer in ectopic breast tissue. Though the tumor cells of apocrine adenocarcinoma and invasive mammary-type ductal carcinoma show different histological features on hematoxylin and eosin stains, they share immunohistochemical results, meaning that an immunohistochemical stain does not help differentiate these diseases [8]. Therefore, it is difficult to distinguish whether two such tumors are of different origin. To exclude the possibility of metastatic mammary apocrine carcinoma, a radiological workup and additional breast examinations, such as breast sonography and mammography, should be performed. In our patient, ectopic mammary tissue was ruled out because of an absence of ectopic mammary tissue in the sample and the tumor in the breast.

According to previous reports, EMPD appears to behave more aggressively when accompanied by an underlying mammarytype carcinoma than when not associated with a mammarytype carcinoma [9]. Since our case was rare, with no established guidelines available, we consulted with various departments, including the pathology and breast surgery departments. Although mammary-type ductal carcinoma was identified in the axilla and immunohistochemical staining showed a result similar to breast cancer, the sample showed no evidence of metasta- sis from apocrine breast carcinoma or breast cancer in ectopic mammary tissue. Therefore, we concluded that this case was a rare adnexal cancer of the skin that was very similar to breast cancer, and we thus treated it according to guidelines for adnexal cancer treatment.

An initial skin biopsy may not provide accurate information because the biopsy only covers superficial lesions. In situations where no information is available regarding the underlying disease, it is advisable to leave a sufficient base safety margin. In the present case, the mass was sufficiently removed with a base safety margin of $1.5 \mathrm{~cm}$. Although no exact guideline has yet been established, it may be advisable to consider surgical resection and reconstruction in light of these characteristics of EMPD.

Many techniques have been used for axillary reconstruction. A skin graft is simplest, but it has the disadvantages of contracture and poor cosmetic results [10]. Other options, such as Z-plasty and the local flap technique, have been used previously. Currently, the regional flap technique is commonly performed because it appears to be superior to other procedures. The latissimus dorsi myocutaneous flap has been used by many surgeons [11]. Although this is a good option, it has the disadvantage of muscle bulkiness, resulting in cosmetic and functional problems [12].

A new technique using a TDAP flap was first described by Angrigiani et al. [13]. Recently, many cases have been reported in which a TDAP flap was used in axillary reconstruction. This technique has the advantage of an improved postoperative range of motion and better cosmetic results [14].

In conclusion, this case of axillary EMPD with underlying invasive apocrine adenocarcinoma and invasive mammary-type ductal carcinoma was successfully treated through wide excision with a sufficient safety margin and axillary reconstruction using a TDAP flap. The only additional treatment that the patient received was adjuvant radiotherapy for the treatment of skin cancer. As mentioned above, in diagnostically challenging EMPD cases such as this one, an investigative workup is essential to exclude invasive ductal carcinoma from ectopic breast tissue and the presence of a metastatic lesion. In addition, because EMPD is usually associated with adnexal cancer, a sufficient safety margin should be provided. Furthermore, this case highlights the need for a multidisciplinary approach to manage these difficult cases. Finally, further studies on this topic should be conducted in the future.

\section{NOTES}

\section{Conflict of interest}

No potential conflict of interest relevant to this article was reported. 


\section{Ethical approval}

The study was performed in accordance with the principles of the Declaration of Helsinki. Written informed consent was obtained.

\section{Patient consent}

The patient provided written informed consent for the publication and the use of her images.

\section{Author contribution}

Writing - original draft: Jang SB. Writing - review \& editing: Jang SB, Kim SE, Kim YA, Choi HR. Approval of final manuscript: all authors.

\section{ORCID}

Seung Bin Jang https://orcid.org/0000-0002-1465-6073

Sung-Eun Kim https://orcid.org/0000-0002-3413-7094

Young Ah Kim https://orcid.org/0000-0001-7850-5509

Hye Ryeon Choi https://orcid.org/0000-0002-5657-5677

\section{REFERENCES}

1. Whorton CM, Patterson JB. Carcinoma of Moll's glands with extramammary Paget's disease of the eyelid. Cancer 1955;8:1009-15.

2. Delport ES. Extramammary Paget's disease of the vulva: an annotated review of the current literature. Australas J Dermatol 2013;54:9-21.

3. Villada G, Farooq U, Yu W, et al. Extramammary Paget disease of the vulva with underlying mammary-like lobular carcinoma: a case report and review of the literature. Am J Dermatopathol 2015;37:295-8.

4. Morgan JM, Carmichael AJ, Ritchie C. Extramammary Paget's disease of the axilla with an underlying apocrine carcinoma. Acta Derm Venereol 1996;76:173-4.
5. Kazakov DV, Spagnolo DV, Kacerovska D, et al. Lesions of anogenital mammary-like glands: an update. Adv Anat Pathol 2011;18:1-28.

6. Jung HR, Kwon SY, Son D. Apocrine carcinoma of the axilla associated with extramammary Paget's disease: a case report and review of the literature. J Pathol Transl Med 2015;49: 535-7.

7. Belousova IE, Kazakov DV, Michal M, et al. Vulvar toker cells: the long-awaited missing link: a proposal for an originbased histogenetic classification of extramammary Paget disease. Am J Dermatopathol 2006;28:84-6.

8. Toledo-Pastrana T, Llombart-Cussac B, Traves-Zapata V, et al. Case report: differential diagnosis between primary cutaneous apocrine adenocarcinoma versus extramammary or metastatic breast adenocarcinoma. Am J Dermatopathol 2014;36:e175-8

9. Ohira S, Itoh K, Osada K, et al. Vulvar Paget's disease with underlying adenocarcinoma simulating breast carcinoma: case report and review of the literature. Int J Gynecol Cancer 2004;14:1012-7.

10. Metaizeau JP, Gayet C, Schmitt M, et al. The use of free full thickness skin grafts in the treatment of complications of burns. Prog Pediatr Surg 1981;14:209-23.

11. Agarwal R, Chandra R. Latissimus dorsi myocutaneous flap reconstruction of neck and axillary burn contractures. Plast Reconstr Surg 2000;106:1216.

12. Wilson IF, Lokeh A, Schubert W, et al. Latissimus dorsi myocutaneous flap reconstruction of neck and axillary burn contractures. Plast Reconstr Surg 2000;105:27-33.

13. Angrigiani C, Grilli D, Siebert J. Latissimus dorsi musculocutaneous flap without muscle. Plast Reconstr Surg 1995; 96:1608-14.

14. Er E, Ucar C. Reconstruction of axillary contractures with thoracodorsal perforator island flap. Burns 2005;31:726-30. 